\title{
Performance Evaluation of some Modulation Techniques for Radio Frequency Identification (RFID) System under different communication channels
}

\author{
Adebayo S. ${ }^{1}$, Ogunti E.O ${ }^{2}$, Awofolaju T.T ${ }^{3}$ \\ ${ }^{1}$ (Computer Science \&Information Technology, Bowen University Iwo,Nigeria,) \\ ${ }^{2}$ (Electrical/Electronic Engineering, Federal University of Technology, Akure Nigeria,) \\ ${ }_{3}^{3}$ (Electrical/Computer Engineering, Afe Babalola UniversityAdo-Ekiti Nigeria,)
}

\begin{abstract}
To transmit data and Radio frequency Identification System (RFID) information from one location to another without physically connecting them together, as in wireless mobility, it is important to choose a suitable modulation scheme. This work presents the design and simulation of RFID using different modulating schemes. Some modulating schemes have been considered which are M-Ary PSK and M-Ary QAM. The study involves using Simulink in MATLAB to design the RFID using the stated modulating schemes as modulating technique. The modulated signals were then passed through additive white Gaussian noise and Rayleigh multipath fading channels to model the transmission of the signals through wireless medium. Each design is optimized to obtain the best design. The various simulations are compared using three figures of merit: Bit Error Rate(BER), Power Efficiency, and Bandwidth efficiency, to determine the best modulating scheme in the design of Radio frequency identification (RFID) system. The result established that QAM has a better performance metrics.
\end{abstract}

Keywords: RFID, PSK, QAM, BER, Power Efficiency, Bandwidth Efficiency, AWGN, Rayleigh Fading Channel

\section{Introduction}

Radio Frequency Identification (RFID) is a term for a small, wireless radio system that uses emitted electromagnetic energy for the purposes of identification. RFID systems consist of four different elements; a transponder (or tag), an interrogator (or reader), an antenna, and a host computer system that acts as both controller and database [1], [2]. The basic system uses a reader that is networked to a host computer system to transmit an interrogation signal through an antenna to a target tag. The tag harnesses the electromagnetic energy and redirects a response through its own antenna back to the reader; thereby "identifying itself". The reader then updates the database as to the presence of the tag in its area of coverage [3]. To transmit data and Radio frequency Identification System (RFID) information from the tag to the reader and vice versa without physically connecting them together, as in wireless mobility, it is important to choose a suitable modulation scheme. A modulation scheme is a process that uses an information signal to alter some properties of a higher frequency carrier waveform. By modulating each information signal into a different carrier frequency, many information signals can be communicated between distant sender and receiver [26]. Radio waves are used as the transmission medium between the sender and receiver. The choice of modulation and demodulation used for the radio communication system is dependent on the required information transfer rate, the available spectrum to convey the information, and the cost [4]-[7]. There are many types of modulation formats used for the transportation of information [8]-[12]. Most digital modulation techniques used in RFID System are classified as either: amplitude shift keying (ASK) modulation, phase shift keying (PSK) modulation or frequency shift keying (FSK) modulation. In ASK, the magnitude of carrier is varied according to the magnitude of message signal. While in PSK and FSK modulations, the angle and the frequency of the carrier are varied with the magnitude of message signal respectively [13]-[16].

In Quadrature Amplitude Modulation (QAM), both the Amplitude and the Phase of the carrier is varied. It is now in use due to the demand of higher bandwidth efficiency [17], especially for data transmission in mobile communications. Multilevel quadracture amplitude modulation (M-QAM) is an attractive modulation technique for wireless communications because of its high spectral efficiency [4], [18]. M-QAM has been recently proposed and studied for various non-adaptive and adaptive wireless systems [19]-[21]. However, the severe amplitude and phase fluctuations inherent to wireless channels significantly degrade the bit-error rate (BER) performance of M-QAM. That is because the demodulator must scale the received signal to normalize channel gain so that its decision regions correspond to the transmitted signal constellation.

PSK is a modulation scheme that conveys data by changing, or modulating, the phase of a reference signal (that is, the phase of the carrier wave is changed to represent the data signal). A finite number of phases are used to represent digital data. Each of these phases is assigned a unique pattern of binary bits; usually each 
phase encodes an equal number of bits. Each pattern of bits forms the symbol that is represented by the particular phase. Owing to PSK's simplicity, particularly when compared to QAM, it is widely used in existing technologies. For example, wireless Local Area Network (LAN) standard, IEEE 802.11b, uses a variety of different PSK's depending on the data rate required [22], [28].

Since the different modulation techniques have different or special attributes that may influence their performance in different applications, this work presents the design and simulation of RFID using different modulating schemes. Some modulating schemes have been considered which are M-Ary PSK, and M-Ary QAM. The study involves using Simulink in MATLAB to design the RFID using the stated modulating schemes as modulating technique. The modulated signals were then passed through additive white Gaussian noise and Rayleigh multipath fading channels to model the transmission of the signals through wireless medium. Each design is optimized to obtain the best design. The various simulations are compared using three figures of merit: Bit Error Rate (BER), Power Efficiency, and Bandwidth efficiency, to determine the best modulating scheme in the design of Radio frequency identification (RFID) system.

\section{Additive White Gaussian Noise (AWGN)}

The simplest type of channel is the Gaussian channel. It is often referred to as the additive white Gaussian noise (AWGN) channel. Basically, it is the noise generated in the receiver side if we assume that the transmitter is ideal and noiseless, thus, AWGN represents the thermal noise generated by electrical instruments. The term additive means the noise is superimposed or added to the signal where it will limit the receiver ability to make correct symbol decisions and limit the rate of information. Thus, AWGN is the effect of thermal noise generated by thermal motion of electron in all dissipative electrical components i.e. resistors, wires and so on. Mathematically, let the bit energy be defined as the ratio of received power to the transmission rate in bits/second. Consider the complex form of the signal envelope. If the power of the signal envelope is normalized to 1 , then the signal energy of each bit is

$$
E_{b}=\int_{0}^{T} P(t) d t=T
$$

where $\mathrm{T}$ is the duration of bit. Consider a noise source

$$
N_{s}=a+j b
$$

where $a$ and $b$ are Gaussian random variables. Since the in-phase and quadrature components of the noise source are independent and have equal power which is 1 , the total noise power is equal to the sum of the power of these components which is 2 . The mean power of the noise is equal $\mathrm{N}_{0} \mathrm{~B}$. where $\mathrm{B}$ is the bandwidth and is equal to the sampling frequency per data symbol $\left(f_{s}\right)$. Thus the noise power spectral density is given as

$$
N_{0}=2 / f_{s}
$$

The sampling rate will be some number $r$ divided by the duration of the bit $(\mathrm{T})$.this will give the sampling frequency $\left(\mathrm{f}_{\mathrm{s}}\right)$ to be

$$
f_{s}=\frac{r}{T}
$$

Substituting for $\mathrm{f}_{\mathrm{s}}$ in equation (2.23) and combining equations 2.21 and 2.23 , we obtain

$$
\frac{E_{b}}{N_{0}}=\frac{r}{2}
$$

Since thermal noise is present in all communication systems and is a prominent noise source for most system, the thermal noise characteristics that are additive, white and Gaussian are most often used to model the noise in communication systems.

\section{Fading channel}

Fading is the direct result of multi-path propagation where radio waves propagate along different paths before arriving at the receiver antenna. These radio waves may arrive at receiver after different delays, with different amplitudes and with different phases. Since signal propagation takes place in the atmosphere and near the ground, apart from the effect of free path loss, the most notable effect of signal degradation is multipath propagation. The effect can cause fluctuations in the received signal's amplitude, phase and angle of arrival, giving rise to multipath fading.

\section{Shannon Equation}

Information theory was invented by Claude Shannon in 1948 to characterize the limits of reliable communication. Before Shannon, it was widely believed that the only way to achieve reliable communication over a noisy channel, that is, to make the error probability as small as desired, was to reduce the data rate. Shannon showed the surprising result that this belief is incorrect: by more intelligent coding of the information, one can, in fact, communicate at a strictly positive rate but at the same time with as small an error probability as 
desired. However, there is a maximal rate, called the capacity of the channel, for which this can be done: if one attempts to communicate at rates above the channel capacity, then it is impossible to drive the error probability to zero. Thus for a system transmitting at maximum capacity, $\mathrm{C}$, the average signal power, $\mathrm{S}$, measured at the receiver input, can be written as

$$
S=E_{b} . C
$$

where $E_{b}$ is the average received energy per bit. The average noise power, $N$, can also be redefined as

$$
N=N_{0} \cdot B
$$

where $\mathrm{N}_{\mathrm{o}}$ is the noise power density (watts/Hz).

Considering all possible multi-level and multi-phase encoding techniques, the Shannon-Hartley theorem states the channel capacity $\boldsymbol{C}$, meaning the theoretical tightest upper bound on the information rate (excluding error correcting codes) of clean (or arbitrarily low bit error rate) data that can be sent with a given average signal power $S$ through an analog communication channel subject to additive white Gaussian noise of power $N$, is:

$$
C=B \log _{2}\left(1+\frac{S}{N}\right)
$$

From equations (2.40) and (2.41), Equation (2.42) which is the Shannon-Hartley theorem can be written in the form

$$
\frac{C}{B}=\log _{2}\left(1+\frac{E_{b} \cdot C}{N_{0} \cdot B}\right)
$$

Thus equation (9) is the Shannon-Hartley capacity limit for error-free communication where $c$ is the channel capacity in bits per second, $\mathrm{B}$ is the bandwidth in $\mathrm{Hz}, \mathrm{S}$ is the received signal power in watts and $\mathrm{N}$ is the noise power.

Equation (9) can be interpreted as follows:

1. $\mathrm{C} / \mathrm{B}$ represents the bandwidth efficiency of the system in bit/second/Hz. The larger the ratio, the greater the bandwidth efficiency.

2. $E_{b} / N_{o}$ is a measure of the power efficiency of the system. The smaller the ratio, the less energy used by each bit (and consequently for each symbol) to be detected successfully in the presence of a given amount of noise. The capacity to transfer error-free information is enhanced with increased bandwidth B, even though the signal-to-noise ratio is decreased because of the increased bandwidth.

\section{System Model}

The proposed RFID system models shown in the Figures 1 were designed in Simulink of MATLAB by referring to the given specification by the International Organization of Standardization (ISO), Electronic Product Code (EPC) global Inc, European Telecommunications Standards Institute (ETSI) and Federal Communications Commission (FCC). Figure 1 shows the block diagram of the model used in this work. The simulation model has a source data, which is the randomly produced data or RFID data. This data is ideal to test the channel impact to the BER performance. The simulation employed gray coding of the generated signal as absolute encoder to prevent data errors. After gray coding, data is mapped from binary data to complex data, and each output datum represents a point in the constellation diagram [23]-[25]. In this model, we use Phase Shift Keying (PSK) Modulation, Quadrature Amplitude Modulation (QAM) and Gaussian minimum shift keying (GMSK) to modulate the data source. The modulated signal was passed through both Additive White Gaussian Noise Channel and Rayleigh Multipath fading Channel. [4], [26]. AWGN channel is very straight forward by just add a white Gaussian noise into signal to meet specified SNR. At the receiver, the received signal was demodulated after passing through a filter. Decision device decide each adjusted symbol, correspondent to each point in constellation, to a binary datum. At last, we get the bit error rate by comparing source data and received binary data. The performance of RFID system is studied based on PSK and QAM modulation techniques that have been used in this simulation.

In mobile wireless communication systems, the channel is time varying because of the motion of either the transmitter or the receiver which results in propagation path changes. Figure 1 shows the configuration of the transmitter and receiver. The data is modulated using the desired modulation scheme using Gray coding. After modulation, the signal is then transmitted over a channel with flat Rayleigh fading and additive white Gaussian noise (AWGN). 


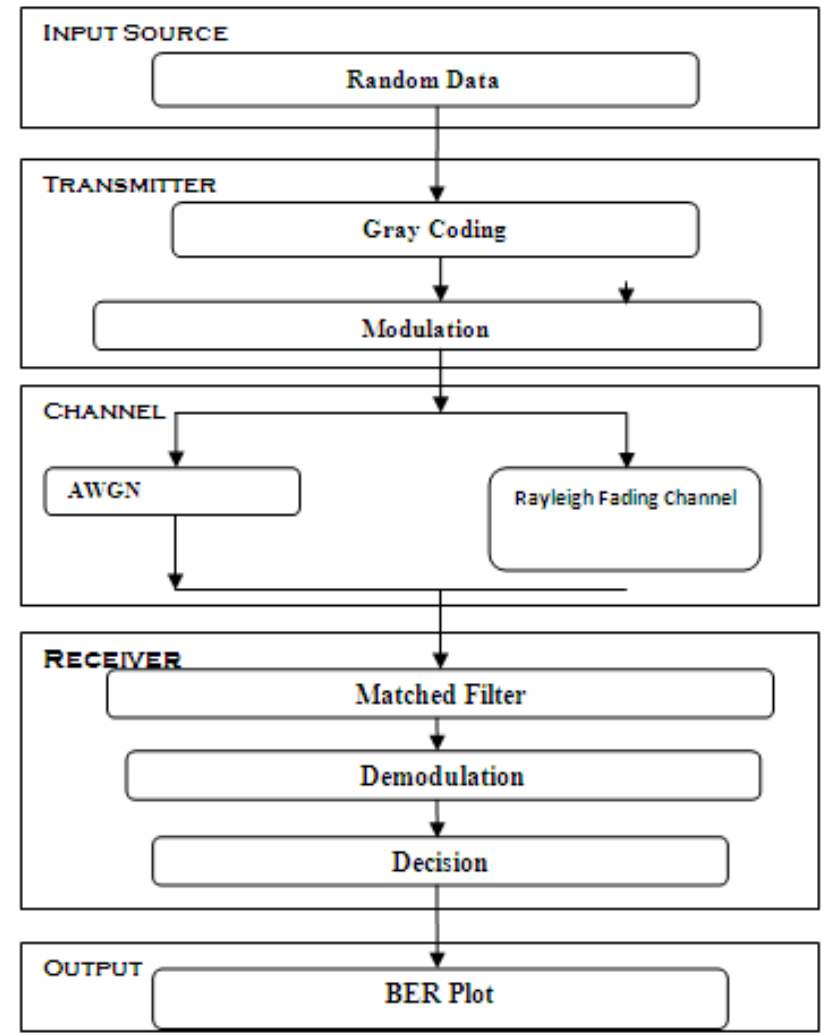

Fig 1 RFID system model (Mohammed et al, 2010)

\section{Simulation Results and Discussion}

In this work, analysis of the performance of RFID System with different modulation schemes in both AWGN and Rayleigh Fading Channel was performed using SIMULINK in MATLAB. Figure 2 shows our SIMULINK model of RFID, while Table 1 shows the simulation parameters for RFID system.

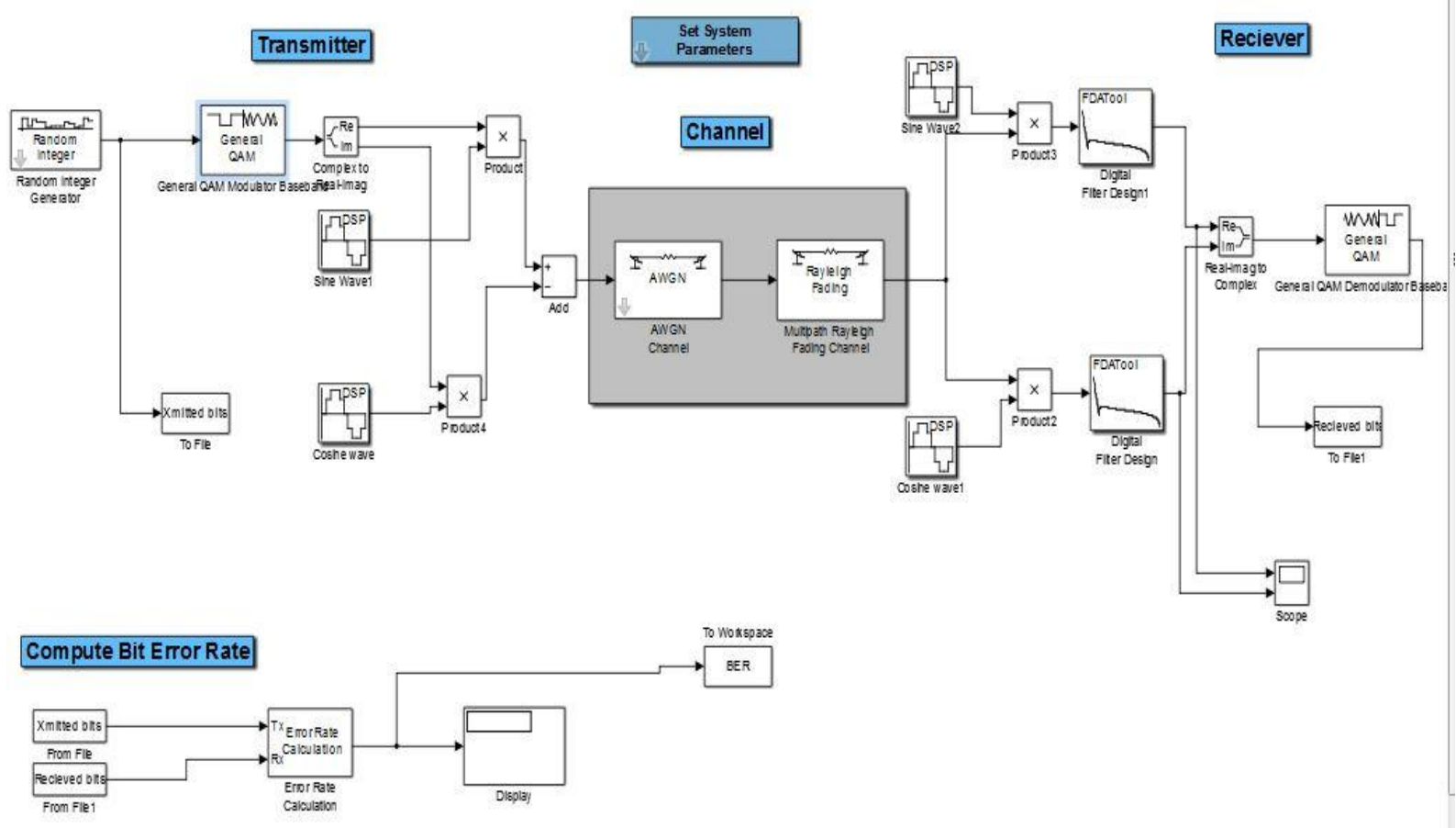

Fig 2 SIMULINK Simulation Model 
Table 1. Simulation Parameters for RFID System

\begin{tabular}{|l|l|l|}
\hline BAND & LOW FREQUENCY & HIGH FREQUENCY \\
\hline Data Rate & $1 \mathrm{~Kb} / \mathrm{s}$ & $25 \mathrm{~Kb} / \mathrm{s}$ \\
\hline Frequency & $134 \mathrm{kHz}$ & $13.56 \mathrm{MHz}$ \\
\hline Modulation used & M-PSK, M-QAM, GMSK & M-PSK, M-QAM, GMSK \\
\hline Channel used & AWGN, Rayleigh Fading & AWGN, Rayleigh Fading \\
\hline Number bits transmitted & $10,000,000$ & $10,000,000$ \\
\hline
\end{tabular}

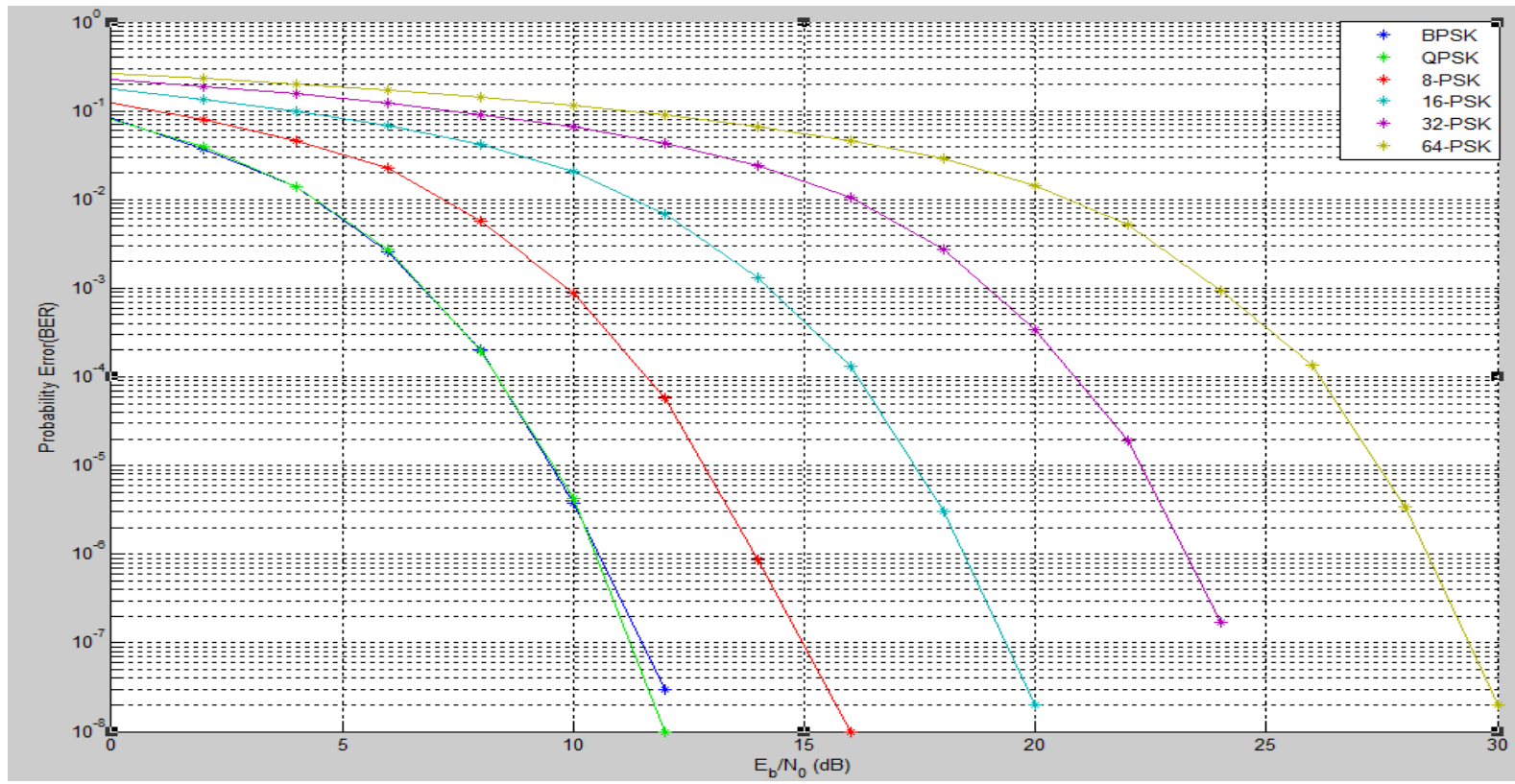

Figure 3 BER versus Signal to Noise Ratio for RFID System with M-Ary PSK modulation in AWGN Channel

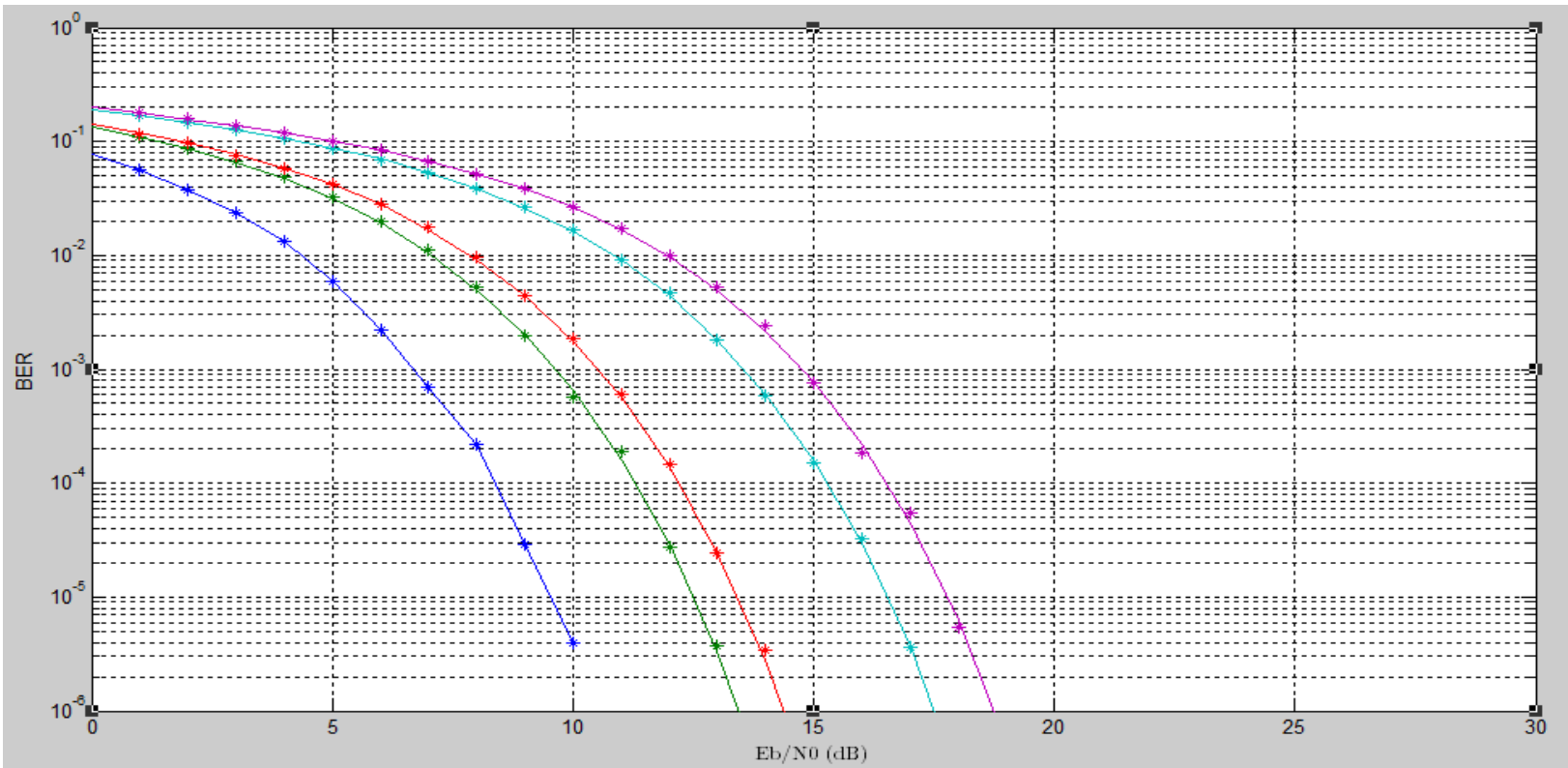

Figure 4 BER versus Signal to Noise Ratio for RFID System with M-Ary QAM modulation in AWGN Channel 


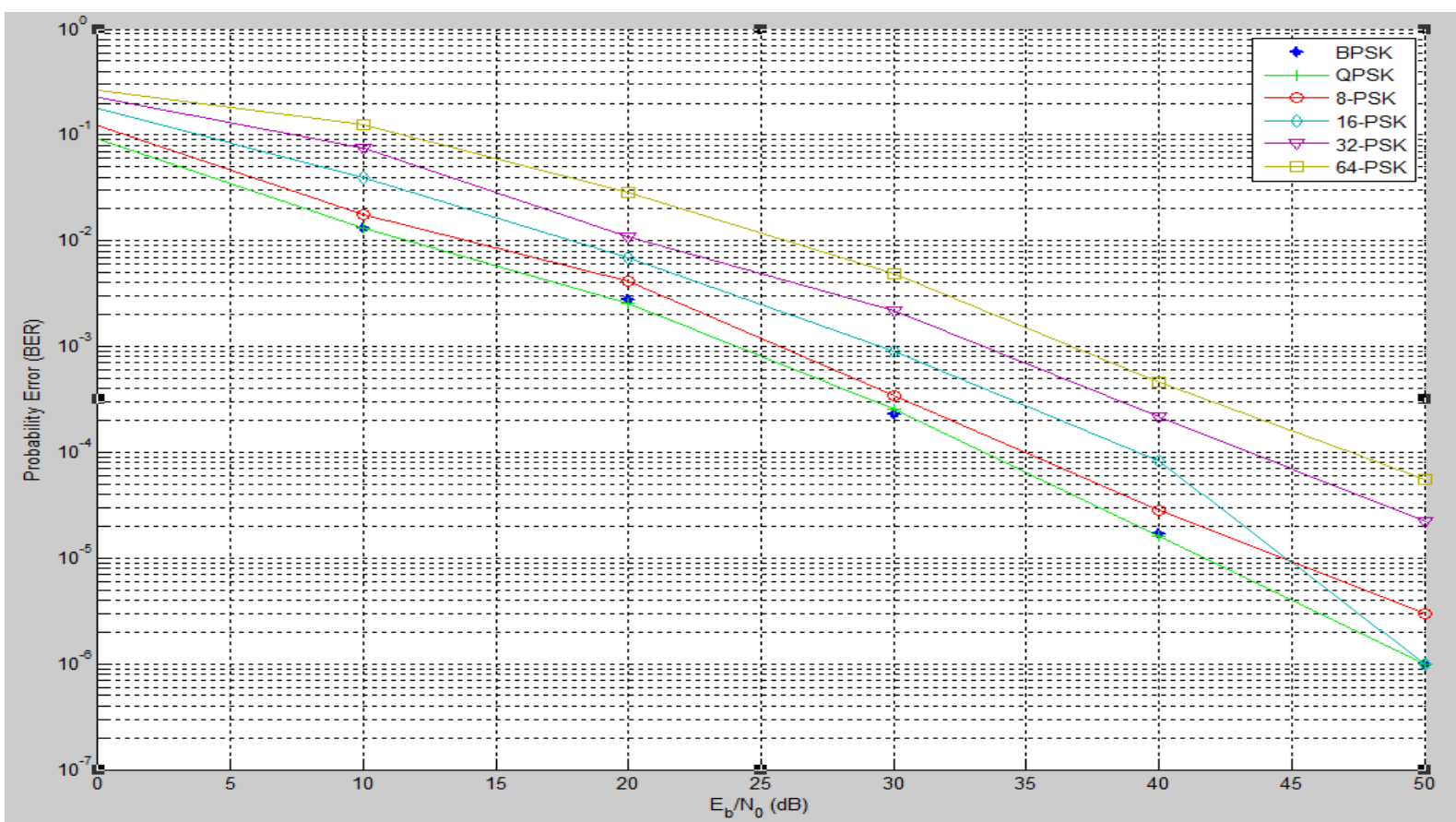

Figure 5 BER versus Signal to Noise Ratio for RFID System with M-Ary PSK modulation in Rayleigh Fading Channel

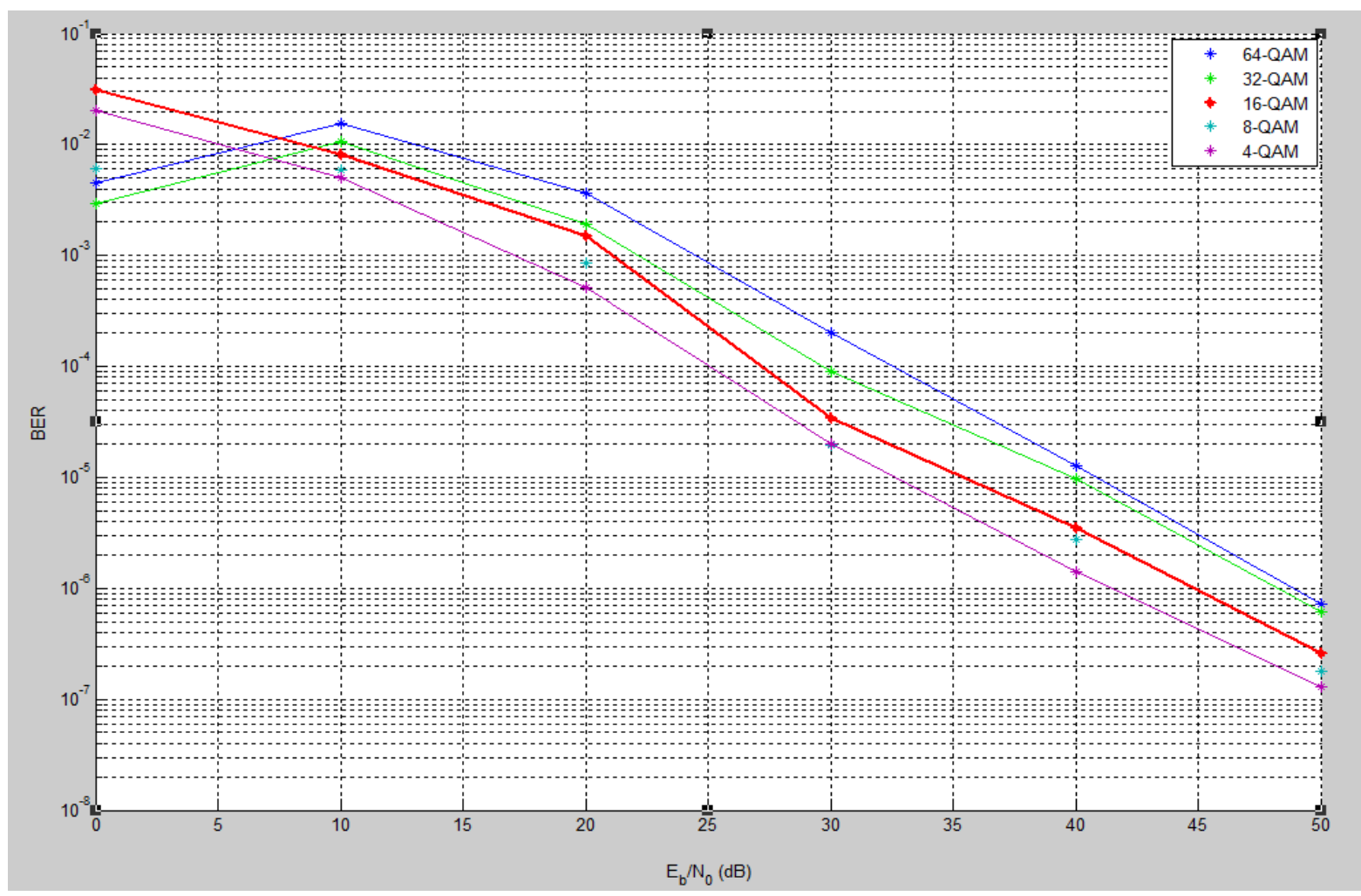

Figure 6 BER versus Signal to Noise Ratio for RFID System with M-Ary QAM modulation in Rayleigh Fading Channel 
Performance Evaluation of some Modulation Techniques for Radio Frequency Identification ...

Table 2 Comparison of RFID System with different modulating schemes at BER of $10^{-6}$

\begin{tabular}{|c|c|c|c|}
\hline Modulating Scheme & $\begin{array}{c}\text { Bandwidth } \\
\text { Efficiency(bit/second/Hz }\end{array}$ & $\begin{array}{c}\mathbf{E}_{\mathbf{b}} / \mathbf{N}_{\mathbf{o}}(\mathbf{d B}) \text { in AWGN } \\
\text { Channel }\end{array}$ & $\begin{array}{c}\mathbf{E}_{\mathbf{b}} / \mathbf{N}_{\mathbf{o}}(\mathbf{d B}) \text { in Rayleigh } \\
\text { Fading Channel }\end{array}$ \\
\hline BPSK & 1 & 11.5 & 50 \\
\hline QPSK & 2 & 11.5 & 50 \\
\hline 4-QAM & 2 & 11.0 & 41 \\
\hline 8-PSK & 3 & 14.0 & 55 \\
\hline 8-QAM & 3 & 13.5 & 45 \\
\hline 16-PSK & 4 & 17.0 & 50 \\
\hline 16-QAM & 4 & 14.0 & 47 \\
\hline 32-PSK & 5 & 22.0 & 60 \\
\hline 32-QAM & 5 & 17.5 & 47 \\
\hline 64-PSK & 6 & 28.0 & 70 \\
\hline 64-QAM & 6 & 19.0 & 48 \\
\hline
\end{tabular}

Fig 3, shows the BER versus the SNR for different levels of PSK modulation Technique in RFID System under AWGN channel. It can be seen that BPSK and QPSK perform better than 8-PSK, 16-PSK, 32-PSK or 64-PSK in terms of power efficiency. Although higher level of PSK Modulation are bandwidth efficient. Fig 4 shows BER versus the SNR for different levels of PSK modulation Technique in RFID System under Rayleigh Fading channel. 8-PSK is more power efficient than all other levels of PSK considered. Fig 5 and Fig 6 shows the BER versus the SNR for different levels of QAM modulation Technique in RFID System under AWGN and Rayleigh Fading channels. It can be seen that 4-QAM is more power efficient than all other levels of QAM. From table 2 it can be concluded that 4-QAM performs better in terms of power efficiency both in AWGN and multipath fading channels when compared to all other modulating schemes that had been considered in this work. It is also more bandwidth efficient that other modulating schemes such as BPSK, QPSK and so on. Thus less energy will be needed to transmit successfully each bit using QAM modulation technique.

\section{Conclusion}

We have studied the performance of RFID with M-PSK and M-QAM over AWGN and Rayleigh multipath fading channels. Comparing the performances of M-PSK and M-QAM in Fig 3 to Fig 6 show that the SNR for RFID with QAM is slightly better than that of RFID with PSK in terms of power efficiency at a BER of $10^{-6}$. However, due to design complexity and cost, QPSK will be favourable.

\section{References}

[1]. A. Kleist, Chapman, T., Sakai, D., \& Jarvis, B. (2004). RFID Labeling - Smart Labeling \& Applications for the Consumer Packaged Goods Supply Chain. Irvine, Ca: Printronix.

[2]. M. Bhuptani, \& Moradpour, S. (2005). RFID Field Guide Deploying Radio Frequency Identification. New York: Prentice Hall.

[3]. S. Garfinkel, \& Rosenberg, B. (2006). RFID Applications, Security and Privacy. New York, New York: Addison Wesley.

[4]. L. Hanzo, R. Steele, and P. Fortune (1990) "A subband coding, BCH coding, and 16-QAM system for mobile radio speech communications," IEEE Trans. Veh. Technol., vol. 39, pp. 327-339.

[5]. J. K. Cavers (1991) "An analysis of pilot symbol assisted modulation for Rayleigh fading channels," IEEE Transactions on Vehicular Technology, vol. VT-40, pp. 686-693, November,.

[6]. S. Sampei and T. Sunaga (1993) "Rayleigh fading compensation for QAM in land mobile radio communications," IEEE Transactions on Vehicular Technology, vol. VT-42, pp. 137-147.

[7]. K. Boulle and J. C. Belfiore (2000) "Modulation schemes designed for the Rayleigh channel," in Pruc. CISS'92, Princeton, NJ, Mar. 1992, pp. 288-293.

[8]. C. Schlegel and D. Costello (1989), "Bandwidth efficient coding for fading channels: Code construction and performance analysis," IEEE J. Select. Areas Commun., vol. 7, pp. 13561368.

[9]. T. S. Rappaport (2004) "Wireless Communications: Principles and Practice", Second Edition,

[10]. H. Jia and D. E. Dodds(2005) "Frame synchronization for PSAM in AWGN and Rayleigh fading channels," Proceedings of IEEE Canadian Conference on Electrical and Computer Engineering, pp. 2290-2296, Saskatoon, May,.

[11]. John Glossner, Daniel Iancu, Jin Lu, Erdem Hokenek, and Mayan Moudgill, Sandbridge Technologies, Inc. ( 2003) “A SoftwareDefined Communications Baseband Design”, IEEE Communications Magazine.

[12]. P. Burns ( 2003) "Software Defined Radio for 3G", Artech.House,.

[13]. J.G.Proakis, M.Salehi (1994) Communication Systems Engineering, Prentice-Hall

[14]. B.P.Lathi, Modern Digital and Analog Communication Systems (1998) 3rd.ed.,Oxford Univ.Press.

[15]. J.G.Proakis (1995) Digital Communications, 3rd.ed., McGraw-Hill.

[16]. S.Benedetto, E.Biglieri, V.Castellani, Digital Transmission Theory, Prentice-.Hall 1987.

[17]. F. Davarian, "Mobile digital communication via tone calibration," IEEE Trans. Veh. Technol., vol. VT-36, pp. 55-62, May 1987.

[18]. G.L.Stüber, Principles of Mobile Communication, Kluwer, 1996

[19]. N. Kinoshita, S. Sampei, E. Moriyama, H. Sasaoka, Y. Kamio, K. Hiramatsu, K. Miya, K. Inogai, and K. Homma, "Field experiments on 16QAM/TDMA and trellis coded 16QAM/TDMA systems for digital land mobile radio communications," Inst. Electron. Commun. Eng. Trans. Commun., vol. E77-B, pp. 911-920, July 1994.

[20]. W. T. Webb and R. Steele, "Variable rate QAM for mobile radio," IEEE Trans. Commun., vol. 43, pp. 2223-2230, July 1995.

[21]. A. Goldsmith and S. G. Chua, "Variable-rate variable-power M-QAM for fading channels," IEEE Trans. Commun., vol. 45, pp. 1218-1230, Oct. 1997.

[22]. Digital Modulation techniques in mobile communications by Fahredd'n Sadikoglu 
[23]. M. Islam, M. A. Hannan, S. A. Samad, A. Hussain. Software Defined Radio for RFID Application, Proceedings of the World Congress on Engineering and Computer Science 2009, (WCECS 2009) Vol I, October 20-22, 2009, San Francisco, USA

[24]. M. Islam, M. A. Hannan, S. A. Samad, A. Hussain. Bit Error Rate (BER) for Modulation Technique Using Software Defined Radio, International Conference of Electrical Engineering and Informatics (ICEEI09), UKM, August 5th-7th, 2009, pp.445-447.

[25]. M. Islam, M. A. Hannan, S. A. Samad, A. Hussain. Modulation Technique for Software Defined Radio Application. Proceedings of the 3rd WSEAS Int. Conf. on Circuits, Systems, Signal and Telecommunications (CISST'09), pp. 179-182 ISSN: 1790-5117.

[26]. [Muhammad Islam, M. A. Hannan, S. A. Samad, A. Hussain (2010). "Performance of RFID with AWGN and Rayleigh Fading Channels for SDR Application." Proceedings of the World Congress on Engineering 2010, vol 1, London, U.K

[27]. J. H. Lodge, M. L. Moher, and S. Crozier, "A comparison of data modulation techniques for land mobile satellite channels," IEEE Trans. Veh. Technol., vol. VT-36, pp. 28-35, Feb. 1987.

[28]. Sam W. Ho, Adaptive Modulation (QPSK, QAM): Intel in Communications, 2004. 